\title{
SOME NEW RESULTS ON BOUNDED SOLUTIONS TO A SEMILINEAR INTEGRO-DIFFERENTIAL EQUATION IN BANACH SPACES
}

\author{
YONG-KUI CHANG, XUE-YAN WEI AND G.M. N'GUÉRÉKATA \\ Communicated by Yuesheng $\mathrm{Xu}$
}

\begin{abstract}
In this article, by a concept of Stepanov type $\mu$-pseudo almost automorphic functions developed recently, we investigate some new existence results on bounded solutions to a semilinear integro-differential equation in Banach spaces. We first establish a new composition theorem of such functions, and then we prove the main results via ergodicity and composition theorems of Stepanov type $\mu$ pseudo almost automorphic functions combined with theories of uniformly exponentially stable and strongly continuous family of operators. These bounded solutions can cover (weighted) pseudo almost automorphic solutions with a Stepanov type forcing term as special cases.
\end{abstract}

1. Introduction. The concept of almost automorphy was first introduced in the literature in relation to some aspects of differential geometry by Bochner in the 1960's [4], which is a natural generalization of almost periodicity; for more details on this topic, we refer to $[\mathbf{1 1}, \mathbf{2 5}, \mathbf{2 6}]$. Since then, this concept has undergone several interesting, natural and powerful generalizations. For example, Liang, Xiao and Zhang in $[\mathbf{2 1}, \mathbf{3 1}]$ presented the concept of pseudo almost automorphy. In [27], N'Guérékata and Pankov introduced the concept of Stepanov-like almost automorphy and applied this concept to study the existence and uniqueness of almost automorphic solutions to the autonomous semilinear equation. Abbas et al. [2] introduced the notation

2010 AMS Mathematics subject classification. Primary 34K14, 35B15, 45N05, $58 \mathrm{D} 25$.

Keywords and phrases. Stepanov type $\mu$-pseudo almost automorphic functions, Integro-differential equations, bounded solutions, fixed point theorems.

This work was supported by NSF of China (No. 11361032) and the Program for Longyuan Youth Innovative Talents of Gansu Province of China (No. 2014-4-80).

Received by the editors on May 2, 2014, and in revised form on December 25, 2014. 
of Stepanov type weighted pseudo almost automorphic sequence and investigated the existence and uniqueness of Stepanov type weighted pseudo almost automorphic sequence solutions to a difference equation. Particularly, Blot, Cieutat and Ezzinbi in [3] applied the measure theory to define a new ergodic function, and they presented the concept of $\mu$-pseudo almost automorphic functions. Chang, N'Guérékata and Zhang presented the notation of Stepanov type $\mu$-pseudo almost automorphic functions and investigated some ergodic properties and composition theorems of Stepanov type $\mu$-pseudo almost automorphic functions in [7].

The above-mentioned concepts have been applied to various differential and integro-differential equations by many scholars; see, for instance, $[6,8,9,10,11,14,15,17,18,20,22,24,32,33,34]$ and the references therein. In particular, Lizama and Ponce [22] systematically studied the existence and uniqueness of bounded solutions, such as almost periodic (automorphic), pseudo-almost periodic (automorphic), asymptotically almost periodic (automorphic), to the following semilinear integro-differential equation:

$$
u^{\prime}(t)=A u(t)+\alpha \int_{-\infty}^{t} e^{-\beta(t-s)} A u(s) d s+f(t, u(t)), \quad t \in \mathbb{R},
$$

where $\alpha, \beta \in \mathbb{R}$ with $\alpha>0, \alpha \neq 0$ and $\alpha+\beta>0, A: D(A) \subseteq \mathbb{X} \rightarrow \mathbb{X}$ is the generator of an immediately norm continuous $C_{0}$-semigroup defined on the Banach space $\mathbb{X}$, and $f: \mathbb{R} \times \mathbb{X} \rightarrow \mathbb{X}$ belongs to a closed subspace of the space of continuous and bounded functions satisfying some Lipschitz type conditions.

Since the concept of Stepanov type $\mu$-pseudo almost automorphic functions has richer information than those of Stepanov type (weighted) pseudo almost automorphic functions, a natural question becomes: what is it about the problem (1.1) when the nonlinear term $f$ is Stepanov type $\mu$-pseudo almost automorphic? To close this gap, the purpose of this article is to deal with the existence of $\mu$-pseudo almost automorphic solutions to problem (1.1) when the forcing term $f: \mathbb{R} \times \mathbb{X} \rightarrow \mathbb{X}$ is a Stepanov type $\mu$-pseudo almost automorphic function presented in [7]. We first give a new composition theorem of such functions (see Theorem 2.28), and then we prove the main results via ergodicity and composition theorems of Stepanov type $\mu$-pseudo almost automorphic functions combined with theories of uniformly 
exponentially stable and strongly continuous family of operators. These bounded solutions can cover (weighted) pseudo almost automorphic solutions with a Stepanov type forcing term as special cases. For more results on different integro-differential equations, please refer to $[1,5,19,23,28,29,30]$ and the references cited therein.

The rest of this paper is organized as follows. In Section 2, we recall some basic definitions, lemmas, and preliminary results which will be used throughout this paper, and a new composition theorem is also proved. In Section 3, we prove the existence of $\mu$-pseudo almost automorphic mild solutions to the equation (1.1).

2. Preliminaries. In this section, we introduce some basic definitions, notation and lemmas which will be used in what follows. In particular, a new composition theorem is established.

Let $(\mathbb{X},\|\cdot\|)$ and $\left(\mathbb{Y},\|\cdot\|_{\mathbb{Y}}\right)$ be two Banach spaces, and let $B C(\mathbb{R}, \mathbb{X})$ denote the Banach space of bounded continuous functions from $\mathbb{R}$ to $\mathbb{X}$, equipped with the supremum norm $\|f\|_{\infty}=\sup _{t \in \mathbb{R}}\|f(t)\|$. The notation $\mathfrak{B}(\mathbb{X}, \mathbb{Y})$ stands for the space of bounded linear operators from $\mathbb{X}$ into $\mathbb{Y}$ endowed uniform operator topology, and we abbreviate to $\mathfrak{B}(\mathbb{X})$, whenever $\mathbb{X}=\mathbb{Y}$. Throughout this work, we denote by $\mathcal{B}$ the Lebesgue $\sigma$-field of $\mathbb{R}$ and by $\mathcal{M}$ the set of all positive measures $\mu$ on $\mathcal{B}$ satisfying $\mu(\mathbb{R})=+\infty$ and $\mu([a, b])<+\infty$, for all $a, b \in \mathbb{R}(a<b)$.

Definition 2.1. [26]. A continuous function $f: \mathbb{R} \rightarrow \mathbb{X}$ is said to be almost automorphic if for every sequence of real numbers $\left\{s_{n}^{\prime}\right\}_{n \in \mathbb{N}}$ there exists a subsequence $\left\{s_{n}\right\}_{n \in \mathbb{N}}$ such that

$$
g(t):=\lim _{n \rightarrow \infty} f\left(t+s_{n}\right)
$$

is well defined for each $t \in \mathbb{R}$, and

$$
\lim _{n \rightarrow \infty} g\left(t-s_{n}\right)=f(t)
$$

for each $t \in \mathbb{R}$. The collection of all such functions will be denoted by $A A(\mathbb{R}, \mathbb{X})$.

Definition 2.2. $[20,26]$. A continuous function $f: \mathbb{R} \times \mathbb{X} \rightarrow \mathbb{X}$ is said to be almost automorphic if $f(t, x)$ is almost automorphic for each 
$t \in \mathbb{R}$ uniformly for all $x \in \mathbb{B}$, where $\mathbb{B}$ is any bounded subset of $\mathbb{X}$. The collection of all such functions will be denoted by $A A(\mathbb{R} \times \mathbb{X}, \mathbb{X})$.

Definition 2.3. [24]. A bounded continuous function with vanishing mean value can be defined as

$$
A A_{0}(\mathbb{R}, \mathbb{X})=\left\{\phi \in B C(\mathbb{R}, \mathbb{X}): \lim _{T \rightarrow \infty} \frac{1}{2 T} \int_{-T}^{T}\|\phi(\sigma)\| d \sigma=0\right\} .
$$

Similarly, we define by $A A_{0}(\mathbb{R} \times \mathbb{Y} \times \mathbb{Y}, \mathbb{X})$ the set of all continuous functions $f: \mathbb{R} \times \mathbb{Y} \times \mathbb{Y} \rightarrow \mathbb{X}$ which belong to $B C(\mathbb{R} \times \mathbb{Y} \times \mathbb{Y}, \mathbb{X})$ and satisfy

$$
\lim _{T \rightarrow \infty} \frac{1}{2 T} \int_{-T}^{T}\|\phi(\sigma, x, y)\| d \sigma=0
$$

uniformly for $(x, y)$ in any bounded subset of $\mathbb{Y} \times \mathbb{Y}$.

Definition 2.4. [3]. Let $\mu \in \mathcal{M}$. A bounded continuous function $f: \mathbb{R} \rightarrow \mathbb{X}$ is said to be $\mu$-ergodic if

$$
\lim _{r \rightarrow+\infty} \frac{1}{\mu([-r, r])} \int_{[-r, r]}\|f(t)\| d \mu(t)=0 .
$$

We denote the space of all such functions by $\epsilon(\mathbb{R}, \mathbb{X}, \mu)$ (or $\epsilon(\mathbb{X}, \mu)$ for abbreviation).

Definition 2.5. [3]. Let $\mu \in \mathcal{M}$. A continuous function $f: \mathbb{R} \times \mathbb{Y} \rightarrow \mathbb{X}$ is said to be $\mu$-ergodic if $f(\cdot, y)$ is bounded for each $y \in \mathbb{Y}$ and

$$
\lim _{r \rightarrow+\infty} \frac{1}{\mu([-r, r])} \int_{[-r, r]}\|f(t, y)\| d \mu(t)=0,
$$

uniformly in $y \in \mathbb{Y}$. We denote the set of all such functions by $\varepsilon(\mathbb{R} \times \mathbb{Y}, \mathbb{X}, \mu)($ or $\epsilon(\mathbb{Y}, \mathbb{X}, \mu)$ for abbreviation).

Definition 2.6. [3]. Let $\mu \in \mathcal{M}$. A continuous function $f: \mathbb{R} \rightarrow \mathbb{X}$ is said to be $\mu$-pseudo almost automorphic if $f$ is written in the form:

$$
f=g+\phi,
$$

where $g \in A A(\mathbb{R}, \mathbb{X})$ and $\phi \in \epsilon(\mathbb{R}, \mathbb{X}, \mu)$. Let $P A A(\mathbb{R}, \mathbb{X}, \mu)$ denote the space of all such functions. 
Definition 2.7. [3]. Let $\mu \in \mathcal{M}$. A continuous function $f: \mathbb{R} \times \mathbb{Y} \rightarrow \mathbb{X}$ is said to be $\mu$-pseudo almost automorphic if $f$ is written in the form:

$$
f=g+\phi
$$

where $g \in A A(\mathbb{R} \times \mathbb{Y}, \mathbb{X})$ and $\phi \in \varepsilon(\mathbb{R} \times \mathbb{Y}, \mathbb{X}, \mu)$.

Lemma 2.8. [3, Proposition 2.13]. Let $\mu \in \mathcal{M}$. Then $\left.\epsilon(\mathbb{R}, \mathbb{X}, \mu),\|\cdot\|_{\infty}\right)$ is a Banach space.

For $\mu \in \mathcal{M}$ and $\tau \in \mathbb{R}$, we denote $\mu_{\tau}$ the positive measure on $(\mathbb{R}, \mathcal{B})$ defined by

$$
\mu_{\tau}(\mathcal{A})=\mu(a+\tau: a \in \mathcal{A}) \quad \text { for } \mathcal{A} \in \mathcal{B}
$$

From $\mu \in \mathcal{M}$, we state the following hypothesis ([3]).

(H0) For all $\tau \in \mathbb{R}$, there exist $\gamma>0$ and a bounded interval $I$ such that

$$
\mu_{\tau}(\mathcal{A}) \leq \gamma \mu(\mathcal{A})
$$

when $\mathcal{A} \in \mathcal{B}$ satisfies $\mathcal{A} \cap I=\varnothing$.

Lemma 2.9. [3]. Let $\mu \in \mathcal{M}$ satisfy $(\mathrm{H} 0)$. Then $\varepsilon(\mathbb{R}, \mathbb{X}, \mu)$ is translation invariant; therefore, $P A A(\mathbb{R}, \mathbb{X}, \mu)$ is also translation invariant.

Lemma 2.10. [3]. Let $\mu \in \mathcal{M}$. Assume that $P A A(\mathbb{R}, \mathbb{X}, \mu)$ is translation invariant. Then the decomposition of a $\mu$-pseudo almost automorphic function in the form $f=g+\phi$ where $g \in A A(\mathbb{R}, \mathbb{X})$ and $\phi \in \varepsilon(\mathbb{R}, \mathbb{X}, \mu)$ is unique.

Lemma 2.11. [3]. Let $\mu \in \mathcal{M}$. Assume that $P A A(\mathbb{R}, \mathbb{X}, \mu)$ is translation invariant. Then $P A A\left(\mathbb{R}, \mathbb{X}, \mu,\|\cdot\|_{\infty}\right)$ is a Banach space.

Definition 2.12. [12, 27]. The Bochner transform $f^{b}(t, s), t \in \mathbb{R}$, $s \in[0,1]$, of a function $f: \mathbb{R} \rightarrow \mathbb{X}$ is defined by

$$
f^{b}(t, s):=f(t+s) .
$$

Definition 2.13. $[\mathbf{1 2}, \mathbf{2 7}]$. Let $p \in[1, \infty)$. The space $B S^{p}(\mathbb{X})$ of all Stepanov bounded functions, with the exponent $p$, consists of all 
measurable functions $f: \mathbb{R} \rightarrow \mathbb{X}$ such that $f^{b} \in L^{\infty}\left(\mathbb{R}, L^{p}(0,1 ; \mathbb{X})\right)$. This is a Banach space with the norm

$$
\|f\|_{S^{p}}=\left\|f^{b}\right\|_{L^{\infty}\left(\mathbb{R}, L^{p}\right)}=\sup _{t \in \mathbb{R}}\left(\int_{t}^{t+1}\|f(\tau)\|^{p} d \tau\right)^{1 / p} .
$$

Definition 2.14. [27]. The space $A S^{p}(\mathbb{X})$ of Stepanov type almost automorphic (or $S^{p}$-almost automorphic) functions consists of all $f \in$ $B S^{p}(\mathbb{X})$ such that $f^{b} \in A A\left(L^{p}(0,1 ; \mathbb{X})\right)$. In other words, a function $f \in L_{l o c}^{p}(\mathbb{R}, \mathbb{X})$ is said to be $S^{p}$-almost automorphic if its Bochner transform $f^{b}: \mathbb{R} \rightarrow L^{p}(0,1 ; \mathbb{X})$ is almost automorphic in the sense that, for every sequence of real numbers $\left\{s_{n}^{\prime}\right\}_{n \in \mathbb{N}}$, there exist a subsequence $\left\{s_{n}\right\}_{n \in \mathbb{N}}$ and a function $g \in L_{\mathrm{loc}}^{p}(\mathbb{R}, \mathbb{X})$ such that

$$
\lim _{n \rightarrow \infty}\left(\int_{t}^{t+1}\left\|f\left(s+s_{n}\right)-g(s)\right\|^{p} d s\right)^{1 / p}=0
$$

and

$$
\lim _{n \rightarrow \infty}\left(\int_{t}^{t+1}\left\|g\left(s-s_{n}\right)-f(s)\right\|^{p} d s\right)^{1 / p}=0
$$

pointwise on $\mathbb{R}$.

Definition 2.15. [27]. A function $f: \mathbb{R} \times \mathbb{Y} \rightarrow \mathbb{X},(t, u) \rightarrow f(t, u)$ with $f(\cdot, u) \in L_{\text {loc }}^{p}(\mathbb{R}, \mathbb{X})$ for each $u \in \mathbb{Y}$, is said to be $S^{p}$-almost automorphic in $t \in \mathbb{R}$ uniformly in $u \in \mathbb{Y}$ if $t \rightarrow f(t, u)$ is $S^{p}$-almost automorphic for each $u \in \mathbb{Y}$. That means, for every sequence of real numbers $\left\{s_{n}^{\prime}\right\}_{n \in \mathbb{N}}$, there exist a subsequence $\left\{s_{n}\right\}_{n \in \mathbb{N}}$ and a function $g(\cdot, u) \in L_{\text {loc }}^{p}(\mathbb{R}, \mathbb{X})$ such that

$$
\lim _{n \rightarrow \infty}\left(\int_{t}^{t+1}\left\|f\left(s+s_{n}, u\right)-g(s, u)\right\|^{p} d s\right)^{1 / p}=0,
$$

and

$$
\lim _{n \rightarrow \infty}\left(\int_{t}^{t+1}\left\|g\left(s-s_{n}, u\right)-f(s, u)\right\|^{p} d s\right)^{1 / p}=0,
$$

pointwise on $\mathbb{R}$ and for each $u \in \mathbb{Y}$. We denote by $A S^{p}(\mathbb{R} \times \mathbb{Y}, \mathbb{X})$ the set of all such functions. 
Definition 2.16. [10]. A function $f \in B S^{p}(\mathbb{X})$ is said to be Stepanov type pseudo almost automorphic if it can be decomposed as $f=g+\varphi$ where $g \in A S^{p}(\mathbb{X})$ and $\varphi^{b} \in A A_{0}\left(\mathbb{R}, L^{p}(0,1 ; \mathbb{X})\right)$. Denote by $P A A^{p}(\mathbb{X})$ the set of all functions.

Definition 2.17. [7]. Let $\mu \in \mathcal{M}$. A function $f \in B S^{p}(\mathbb{X})$ is said to be Stepanov type $\mu$-pseudo almost automorphic (or $S^{p}$ - $\mu$-pseudo almost automorphic) if it can be expressed as $f=g+\phi$, where $g \in A S^{p}(\mathbb{X})$ and $\phi^{b} \in \epsilon\left(L^{p}(0,1 ; \mathbb{X}), \mu\right)$. In other words, a function $f \in L_{\text {loc }}^{p}(\mathbb{R}, \mathbb{X})$ is said to be Stepanov type $\mu$-pseudo almost automorphic relatively to the measure $\mu$, if its Bochner transform $f^{b}: \mathbb{R} \rightarrow L^{p}(0,1 ; \mathbb{X})$ is $\mu$-pseudo almost automorphic in the sense that there exist two functions $g, \phi: \mathbb{R} \rightarrow \mathbb{X}$ such that $f=g+\phi$, where $g \in A S^{p}(\mathbb{X})$ and $\phi^{b} \in \epsilon\left(L^{p}(0,1 ; \mathbb{X}), \mu\right)$, that is, $\phi^{b} \in B C\left(L^{p}(0,1 ; \mathbb{X})\right)$ and

$$
\lim _{r \rightarrow \infty} \frac{1}{\mu([-r, r])} \int_{[-r, r]}\left(\int_{t}^{t+1}\|\phi(s)\|^{p} d s\right)^{1 / p} d \mu(t)=0 .
$$

The set of all such functions will be denoted by $P A A^{p}(\mathbb{R}, \mathbb{X}, \mu)$.

Definition 2.18. [7]. Let $\mu \in \mathcal{M}$. A function $f: \mathbb{R} \times \mathbb{Y} \rightarrow \mathbb{X}$, $(t, u) \rightarrow f(t, u)$ with $f(\cdot, u) \in L_{\text {loc }}^{p}(\mathbb{R}, \mathbb{X})$ for each $u \in \mathbb{Y}$, is said to be Stepanov type $\mu$-pseudo almost automorphic (or $S^{p}$ - $\mu$-pseudo almost automorphic) if it can be expressed as $f=g+\phi$, where $g \in A S^{p}(\mathbb{R} \times \mathbb{Y}, \mathbb{X})$ and $\phi^{b} \in \epsilon\left(\mathbb{Y}, L^{p}(0,1 ; \mathbb{X}), \mu\right)$. We denote by $P A A^{p}(\mathbb{R} \times \mathbb{Y}, \mathbb{X}, \mu)$ the set of all such functions.

Lemma 2.19. [7]. Let $\mu \in \mathcal{M}$ and $I$ be a bounded interval (eventually $I=\emptyset)$. Assume that $f(\cdot) \in B S^{p}(\mathbb{R}, \mathbb{X})$. Then the following assertions are equivalent:

(i) $f^{b}(\cdot) \in \epsilon\left(L^{p}(0,1 ; \mathbb{X}), \mu\right)$.

(ii) $\lim _{r \rightarrow+\infty} \frac{1}{\mu([-r, r] \backslash I)} \int_{[-r, r] \backslash I}\left(\int_{t}^{t+1}\|f(s)\|^{p} d s\right)^{1 / p} d \mu(t)=0$.

(iii) For any $\varepsilon>0$,

$$
\lim _{r \rightarrow+\infty} \frac{\mu\left(\left\{t \in[-r, r] \backslash I:\left(\int_{t}^{t+1}\|f(s)\|^{p} d s\right)^{1 / p}>\varepsilon\right\}\right)}{\mu([-r, r] \backslash I}=0 .
$$


Lemma 2.20. [7]. Let $\mu \in \mathcal{M}$ satisfy $(\mathrm{H} 0)$. Then $\epsilon\left(L^{p}(0,1 ; \mathbb{X}), \mu\right)$ is translation invariant; therefore, $P A A^{p}(\mathbb{R}, \mathbb{X}, \mu)$ is also translation invariant.

Lemma 2.21. [7]. Let $\mu \in \mathcal{M}$ satisfy (H0). If $f \in P A A(\mathbb{R}, \mathbb{X}, \mu)$, then $f \in P A A^{p}(\mathbb{R}, \mathbb{X}, \mu)$ for each $1 \leq p<\infty$. In other words, $P A A(\mathbb{R}, \mathbb{X}, \mu) \subseteq P A A^{p}(\mathbb{R}, \mathbb{X}, \mu)$.

Thus, we have

$$
A A(\mathbb{R}, \mathbb{X}) \subset P A A(\mathbb{R}, \mathbb{X}, \mu) \subset P A A^{p}(\mathbb{R}, \mathbb{X}, \mu) .
$$

Lemma 2.22. [7]. Let $\mu \in \mathcal{M}$ and $f \in P A A^{p}(\mathbb{R}, \mathbb{X}, \mu)$ be such that $f=g+\chi$, where $g \in A S^{p}(\mathbb{X})$ and $\chi^{b} \in \epsilon\left(L^{p}(0,1 ; \mathbb{X}), \mu\right)$. If $P A A^{p}(\mathbb{R}, \mathbb{X}, \mu)$ is translation invariant, then

$$
\{g(t): t \in \mathbb{R}\} \subseteq \overline{\{f(t): t \in \mathbb{R}\}} \text {, (the closure of range } f \text { ). }
$$

Lemma 2.23. [7]. Let $\mu \in \mathcal{M}$. Assume that $P A A^{p}(\mathbb{R}, \mathbb{X}, \mu)$ is translation invariant. Then $\left(P A A^{p}(\mathbb{R}, \mathbb{X}, \mu),\|\cdot\|_{S^{p}}\right)$ is a Banach space.

Lemma 2.24. [7]. Let $\mu \in \mathcal{M}$. Assume that $P A A^{p}(\mathbb{R}, \mathbb{X}, \mu)$ is translation invariant. Then the decomposition of an $S^{p}-\mu$-pseudo almost automorphic function in the form $f=g+\chi$, where $g \in A S^{p}(\mathbb{X})$ and $\chi^{b} \in \epsilon\left(L^{p}(0,1 ; \mathbb{X}), \mu\right)$ is unique.

Lemma 2.25. [7]. Let $\mu \in \mathcal{M}$. Suppose that $f=g+h \in P A A^{p}(\mathbb{R} \times$ $\mathbb{X}, \mathbb{X}, \mu)$ with $g \in A S^{p}(\mathbb{R} \times \mathbb{X}, \mathbb{X}), h^{b} \in \epsilon\left(\mathbb{X}, L^{p}(0,1 ; \mathbb{X}), \mu\right)$ and satisfies the following condition:

(H1) There exists a constant $L>0$ such that, for all $x, y \in \mathbb{X}$ and $t \in \mathbb{R}$

$$
\|f(t, x)-f(t, y)\| \leq L\|x-y\| .
$$

If $v=v_{1}+v_{2} \in P A A^{p}(\mathbb{R}, \mathbb{X}, \mu)$ with $v_{1} \in A S^{p}(\mathbb{X}), v_{2}^{b} \in \epsilon\left(L^{p}(0,1 ; \mathbb{X}), \mu\right)$ and $K_{1}=\overline{\left\{v_{1}(t) ; t \in \mathbb{R}\right\}}$ is compact. Then $f(\cdot, v(\cdot)) \in P A A^{p}(\mathbb{R}, \mathbb{X}, \mu)$.

Lemma 2.26. [7]. Let $\mu \in \mathcal{M}$ and $f=g+h \in P A A^{p}(\mathbb{R} \times \mathbb{X}, \mathbb{X}, \mu)$ with $g \in A S^{p}(\mathbb{R} \times \mathbb{X}, \mathbb{X}), h^{b} \in \epsilon\left(\mathbb{X}, L^{p}(0,1 ; \mathbb{X}), \mu\right)$. Assume that the following conditions are satisfied: 
(i) there exists a nonnegative function $L(\cdot) \in B S^{p}(\mathbb{R})$ with $p>1$ such that, for all $x, y \in \mathbb{X}$ and $t \in \mathbb{R}$,

$$
\begin{aligned}
& \left(\int_{t}^{t+1}\|f(s, x)-f(s, y)\|^{p} d s\right)^{1 / p}<L(t)\|x-y\|, \\
& \lim _{r \rightarrow \infty} \frac{1}{\mu([-r, r])} \int_{[-r, r]} L(t) d \mu(t)<\infty .
\end{aligned}
$$

(ii) $g(t, x)$ is uniformly continuous in any bounded subset $K^{\prime} \subseteq \mathbb{X}$ uniformly for $t \in \mathbb{R}$. If $u=u_{1}+u_{2} \in P A A^{p}(\mathbb{R}, \mathbb{X}, \mu)$, with $u_{1} \in A S^{p}(\mathbb{X}), u_{2}^{b} \in \epsilon\left(L^{p}(0,1 ; \mathbb{X}), \mu\right)$ and $K_{2}=\overline{\left\{u_{1}(t): t \in \mathbb{R}\right\}}$ is compact, then $f(\cdot, u(\cdot))$ belongs to $P A A^{p}(\mathbb{R}, \mathbb{X}, \mu)$.

Lemma 2.27. [7]. Let $\mu \in \mathcal{M}$ and $f=g+\phi \in P A A^{p}(\mathbb{R} \times \mathbb{X}, \mathbb{X}, \mu)$ with $g \in A S^{p}(\mathbb{R} \times \mathbb{X}, \mathbb{X}), \phi^{b} \in \epsilon\left(\mathbb{X}, L^{p}(0,1 ; \mathbb{X}), \mu\right)$. Assume that following conditions hold:

(i) $f(t, x)$ is uniformly continuous in any bounded subset $K^{\prime} \subseteq \mathbb{X}$ uniformly for $t \in \mathbb{R}$,

(ii) $g(t, x)$ is uniformly continuous in any bounded subset $K^{\prime} \subseteq \mathbb{X}$ uniformly for $t \in \mathbb{R}$,

(iii) For any bounded subset $K^{\prime} \subseteq \mathbb{X},\left\{f(\cdot, x): x \in K^{\prime}\right\}$ is bounded in $P A A^{p}(\mathbb{R} \times \mathbb{X}, \mathbb{X}, \mu)$. If $x=v_{1}+v_{2} \in P A A^{p}(\mathbb{R}, \mathbb{X}, \mu)$, with $v_{1} \in A S^{p}(\mathbb{X}), v_{2}^{b} \in \epsilon\left(L^{p}(0,1 ; \mathbb{X}), \mu\right)$ and $Q=\overline{\{x(t): t \in \mathbb{R}\}}, Q_{1}=$ $\overline{\left\{v_{1}(t): t \in \mathbb{R}\right\}}$ are compact, then $f(\cdot, x(\cdot))$ belongs to $P A A^{p}(\mathbb{R}, \mathbb{X}, \mu)$.

Based upon [13, Theorem 2.4] and [7, Theorem 3.1], we have the following composition theorem for Stepanov type $\mu$-pseudo almost automorphic functions.

Theorem 2.28. Let $\mu \in \mathcal{M}, p>1$ and $f=g+\chi \in P A A^{p}(\mathbb{R} \times \mathbb{X}, \mathbb{X}, \mu)$ with $g \in A S^{p}(\mathbb{R} \times \mathbb{X}, \mathbb{X}), \chi^{b} \in \epsilon\left(\mathbb{X}, L^{p}(0,1 ; \mathbb{X}), \mu\right)$. Assume that the following conditions are satisfied:

(i) there exist nonnegative functions $\mathcal{L}_{f}(\cdot), \mathcal{L}_{g}(\cdot) \in A S^{\mathrm{r}}(\mathbb{R}, \mathbb{R})$ with $\mathbb{r} \geq \max \left\{p, \frac{p}{p-1}\right\}$ such that, for all $u, v \in \mathbb{X}$ and $t \in \mathbb{R}$,

$$
\begin{aligned}
& \|f(s, u)-f(s, v)\| \leq \mathcal{L}_{f}(t)\|u-v\|, \\
& \|g(s, u)-g(s, v)\| \leq \mathcal{L}_{g}(t)\|u-v\| ;
\end{aligned}
$$


(ii) $u=u_{1}+u_{2} \in P A A^{p}(\mathbb{R}, \mathbb{X}, \mu)$, with $u_{1} \in A S^{p}(\mathbb{X}), u_{2}^{b} \in$ $\epsilon\left(L^{p}(0,1 ; \mathbb{X}), \mu\right)$ and $K_{3}=\overline{\left\{u_{1}(t): t \in \mathbb{R}\right\}}$ is compact in $\mathbb{X}$.

Then there exists $q \in[1, p)$ such that $\digamma: \mathbb{R} \rightarrow \mathbb{X}$ defined by $\digamma(\cdot)=$ $f(\cdot, u(\cdot))$ belongs to $P A A^{q}(\mathbb{R}, \mathbb{X}, \mu)$.

Proof. Let us decompose $f$ as

$$
\begin{aligned}
f(t, u(t)) & =g\left(t, u_{1}(t)\right)+f(t, u(t))-g\left(t, u_{1}(t)\right) \\
& =g\left(t, u_{1}(t)\right)+f(t, u(t))-f\left(t, u_{1}(t)\right)+\chi\left(t, u_{1}(t)\right),
\end{aligned}
$$

and set

$$
\begin{aligned}
G(t) & =g\left(t, u_{1}(t)\right), \\
F(t) & =f(t, u(t))-f\left(t, u_{1}(t)\right), \\
H(t) & =\chi\left(t, u_{1}(t)\right) .
\end{aligned}
$$

Then $f(t, u(t))=G(t)+F(t)+H(t)$. Since $\mathbb{r} \geq \frac{p}{p-1}$, there exists $q \in[1, p)$ such that $\mathrm{r}=\frac{p q}{p-q}$. Let $p^{\prime}=\frac{p}{p-q}, q^{\prime}=\frac{p}{q}$. Then $p^{\prime}, q^{\prime}>1$ and $\frac{1}{p^{\prime}}+\frac{1}{q^{\prime}}=1$. In view of assumptions (i)-(ii) and [13, Theorem 2.4], we have $g\left(t, u_{1}(t)\right) \in A S^{q}(\mathbb{X})$. The remainder of the proof is to show $F^{b}(t), H^{b}(t) \in \epsilon\left(L^{q}(0,1 ; \mathbb{X}), \mu\right)$, which can be conducted similarly as [7, Theorem 3.1] noting that $\frac{1}{p^{\prime}}+\frac{1}{q^{\prime}}=1$. We omit the details here. The proof is completed.

Next, we recall some recent results from [22] on uniform exponential stability of solutions to the following abstract homogeneous Volterra equation:

$$
\left\{\begin{array}{l}
u^{\prime}(t)=A u(t)+\alpha \int_{0}^{t} e^{-\beta(t-s)} A u(s) d s, \quad t \geq 0 \\
u(0)=x
\end{array}\right.
$$

A solution of equation (2.1) is said to be uniformly exponentially bounded if, for some $\omega \in \mathbb{R}$, there exists a constant $M>0$ such that, for each $x \in D(A)$, the corresponding solution $u(t)$ satisfies

$$
\|u(t)\| \leqslant M e^{-\omega t}, \quad t \geqslant 0 .
$$

In particular, we say that the solutions of equation (2.1) are uniformly exponentially stable if condition (2.2) holds for some $\omega>0$ and $M>0$. 
Definition 2.29. [22, Definition 2.3]. Let $\mathbb{X}$ be a Banach space. A strongly continuous function $T: \mathbb{R}_{+} \rightarrow \mathfrak{B}(\mathbb{X})$ is said to be immediately norm continuous if $T:(0, \infty) \rightarrow \mathfrak{B}(\mathbb{X})$ is continuous.

Lemma 2.30. [22, Theorem 2.4]. Let $\beta>0, \alpha \neq 0$ and $\alpha+\beta>0$ be given. Assume that:

(A1) A generates an immediately norm continuous $C_{0}$-semigroup on a Banach space $\mathbb{X}$

$(\mathrm{A} 2) \sup \left\{\mathfrak{K} \lambda, \lambda \in \mathbb{C}: \lambda(\lambda+\beta)(\lambda+\alpha+\beta)^{-1} \in \sigma(A)\right\}<0$.

Then, the solutions of the problem (2.1) are uniformly exponentially stable.

Lemma 2.31. [22, Proposition 3.1]. Let $\beta>0, \alpha \neq 0$ and $\alpha+\beta>0$. Assume that conditions (A1) and (A2) in Lemma 2.30 hold. Then there exists a uniformly exponentially stable and strongly continuous family of operators $\{S(t)\}_{t \geqslant 0} \subset \mathfrak{B}(\mathbb{X})$ such that $S(t)$ commutes with $A$, that is, $S(t) D(A) \subset D(A), A S(t) x=S(t) A x$ for all $x \in D(A), t \geqslant 0$, and

$$
S(t) x=x+\int_{0}^{t} b(t-s) A S(s) x d s, \quad \text { for all } x \in \mathbb{X}, t \geqslant 0,
$$

where $b(t):=1+\frac{\alpha}{\beta}\left[1-e^{-\beta t}\right], t \geq 0$.

Now, we list a useful compactness criterion.

Let $\varrho(\cdot): \mathbb{R} \rightarrow \mathbb{R}$ be a continuous function such that $\varrho(t) \geq 1$ for all $t \in \mathbb{R}$ and $\varrho(t) \rightarrow \infty$ as $|t| \rightarrow \infty$. We consider the space

$$
C_{\varrho}(\mathbb{X})=\left\{u \in C(\mathbb{R}, \mathbb{X}): \lim _{|t| \rightarrow \infty} \frac{u(t)}{\varrho(t)}=0\right\} .
$$

Endowed with the norm $\|u\|_{\varrho}=\sup _{t \in \mathbb{R}} \frac{\|u(t)\|}{\varrho(t)}$, it is a Banach space (see [18]).

Lemma 2.32. [18]. A subset $\Xi \subseteq C_{\varrho}(\mathbb{X})$ is a relatively compact set if it verifies the following conditions: $(\mathrm{C} 1)$ The set $\Xi(t)=\{u(t): u \in \Xi\}$ is relatively compact in $\mathbb{X}$ for each $t \in \mathbb{R}$.

(C2) The set $\Xi$ is equicontinuous.

(C3) For each $\varepsilon>0$, there exists $\mathbb{L}>0$ such that $\|u(t)\| \leq \varepsilon \varrho(t)$ for all $u \in \Xi$ and all $|t|>\mathbb{L}$. 
Lemma 2.33. [16] (Leray-Schauder alternative theorem). Let $\mathbb{D}$ be a closed convex subset of a Banach space $\mathbb{X}$ such that $0 \in \mathbb{D}$. Let $\Gamma: \mathbb{D} \rightarrow \mathbb{D}$ be a completely continuous map. Then the set $\{x \in \mathbb{D}: x=$ $\lambda \Gamma(x), 0<\lambda<1\}$ is unbounded or the map $\Gamma$ has a fixed point in $\mathbb{D}$.

3. Main results. In this section, we consider the existence of $\mu$ pseudo almost automorphic mild solutions for the problem (1.1) with Stepanov type $\mu$-pseudo almost automorphic forcing term $f$ under some suitable conditions.

Definition 3.1. [22]. A function $u: \mathbb{R} \rightarrow \mathbb{X}$ is said to be a mild solution to equation (1.1) if

$$
u(t)=\int_{\infty}^{t} S(t-s) f(s, u(s)) d s
$$

for all $t \in \mathbb{R}$, where $\{S(t)\}_{t \geqslant 0}$ is given in Lemma 2.31.

First, we list the following basic assumptions:

(T1) For strongly continuous functions $S:[0, \infty) \rightarrow \mathfrak{B}(\mathbb{X})$, there exist $\omega>0, M>0$ such that $\|S(t)\| \leq M e^{-\omega t}$ for all $t \in \mathbb{R}_{+}$.

(T2) Assume that $f \in P A A^{p}(\mathbb{R} \times \mathbb{X}, \mathbb{X}, \mu)$ and there exists a positive number $\mathbb{L}_{f}$ such that

$$
\|f(t, x)-f(t, y)\| \leq \mathbb{L}_{f}\|x-y\|
$$

for all $t \in \mathbb{R}$ and each $x, y \in \mathbb{X}$.

(T3) Suppose that $f \in P A A^{p}(\mathbb{R} \times \mathbb{X}, \mathbb{X}, \mu)$ and there exists a nonnegative function $L_{f}(\cdot) \in B S^{p}(\mathbb{R})$, with $p>1$ such that

$$
\begin{gathered}
\|f(t, x)-f(t, y)\| \leq L_{f}(t)\|x-y\|, \\
\lim _{r \rightarrow \infty} \frac{1}{\mu([-r, r])} \int_{[-r, r]} L_{f}(t) d \mu(t)<\infty,
\end{gathered}
$$

for all $t \in \mathbb{R}$ and each $x, y \in \mathbb{X}$.

(T4) The function $f=g+h \in P A A^{p}(\mathbb{R} \times \mathbb{X}, \mathbb{X}, \mu)$ with $g \in A S^{p}(\mathbb{R} \times$ $\mathbb{X}, \mathbb{X}), h^{b} \in \epsilon\left(\mathbb{X}, L^{p}(0,1 ; \mathbb{X}), \mu\right)$, and there exist nonnegative functions $\mathcal{L}_{f}(\cdot), \quad \mathcal{L}_{g}(\cdot) \in A S^{\mathbb{r}}(\mathbb{R}, \mathbb{R})$ with $\mathbb{r} \geq \max \left\{p, \frac{p}{p-1}\right\}$ such that for all 
$u, v \in \mathbb{X}$ and $t \in \mathbb{R}$,

$$
\begin{gathered}
\|f(s, u)-f(s, v)\| \leq \mathcal{L}_{f}(t)\|u-v\|, \\
\|g(s, u)-g(s, v)\| \leq \mathcal{L}_{g}(t)\|u-v\| .
\end{gathered}
$$

(T5) The function $f=g+h \in P A A^{p}(\mathbb{R} \times \mathbb{X}, \mathbb{X}, \mu)$ where $g \in$ $A S^{p}(\mathbb{R} \times \mathbb{X}, \mathbb{X})$ is uniformly continuous in any bounded subset $\mathbb{M} \subset \mathbb{X}$ uniformly in $t \in \mathbb{R}$ and $h^{b} \in \epsilon\left(\mathbb{X}, L^{p}(0,1 ; \mathbb{X}), \mu\right)$.

(T6) $f \in P A A^{p}(\mathbb{R} \times \mathbb{X}, \mathbb{X}, \mu)$ and $f(t, x)$ is uniformly continuous in any bounded subset $\mathbb{M} \subset \mathbb{X}$ uniformly for $t \in \mathbb{R}$ and for every bounded subset $\mathbb{M} \subset \mathbb{X},\{f(\cdot, x): x \in \mathbb{M}\}$ is bounded in $P A A^{p}(\mathbb{R} \times \mathbb{X}, \mathbb{X}, \mu)$.

Lemma 3.2. Let $\mu \in \mathcal{M}$ and $\beta>0, \alpha \neq 0$ with $\alpha+\beta>0$ and conditions (A1)-(A2) in Lemma 2.30 hold. Assume that (T1) is satisfied. If $f: \mathbb{R} \rightarrow \mathbb{X}$ is Stepanov type $\mu$-pseudo almost automorphic, and $F(t)$ is given by

$$
F(t)=\int_{-\infty}^{t} S(t-s) f(s) d s, \quad t \in \mathbb{R},
$$

then $F \in P A A(\mathbb{R}, \mathbb{X}, \mu)$.

Proof. The proof of uniqueness is similar to [32]. Now let us investigate the existence. Since $f \in P A A^{p}(\mathbb{R}, \mathbb{X}, \mu)$, there exist $g_{1} \in$ $A S^{p}(\mathbb{X})$ and $g_{2}^{b} \in \epsilon\left(L^{p}(0,1 ; \mathbb{X}), \mu\right)$ such that $f=g_{1}+g_{2}$. So

$$
\begin{aligned}
F(t)= & \int_{-\infty}^{t} S(t-s) g_{1}(s) d s \\
& +\int_{-\infty}^{t} S(t-s) g_{2}(s) d s \\
= & \phi(t)+\psi(t),
\end{aligned}
$$

where $\phi(t)=\int_{-\infty}^{t} S(t-s) g_{1}(s) d s$, and $\psi(t)=\int_{-\infty}^{t} S(t-s) g_{2}(s) d s$. We just need to verify $\phi(t) \in A A(\mathbb{X})$ and $\psi(t) \in \epsilon(\mathbb{R}, \mathbb{X}, \mu)$. First we prove that $\phi(t) \in A A(\mathbb{X})$. It follows from [11, Lemma 11.2] that $\phi(t)$ is almost automorphic. Next, we prove that $\psi(t) \in \epsilon(\mathbb{R}, \mathbb{X}, \mu)$. For this, we consider

$$
\psi_{n}(t)=\int_{t-n}^{t-n+1} S(t-s) g_{2}(s) d s,
$$


for each $t \in \mathbb{R}$ and $n=1,2,3, \ldots$ From assumption (T1) and Hölder's inequality, it follows that

$$
\begin{aligned}
\left\|\psi_{n}(t)\right\| & \leq M \int_{t-n}^{t-n+1} e^{-\omega(t-s)}\left\|g_{2}(s)\right\| d s \\
& \leq M\left(\int_{t-n}^{t-n+1} e^{-q \omega(t-s)} d s\right)^{1 / q}\left(\int_{t-n}^{t-n+1}\left\|g_{2}(s)\right\|^{p} d s\right)^{1 / p} \\
& \leq M\left(\int_{n-1}^{n} e^{-q \omega s} d s\right)^{1 / q}\left(\int_{t-n}^{t-n+1}\left\|g_{2}(s)\right\|^{p} d s\right)^{1 / p} \\
& \leq \frac{M}{\sqrt[q]{q \omega}}\left(e^{-q \omega(n-1)}-e^{-q \omega n}\right)^{1 / q}\left(\int_{t-n}^{t-n+1}\left\|g_{2}(s)\right\|^{p} d s\right)^{1 / p} \\
& \leq \frac{M e^{-\omega n}}{\sqrt[q]{q \omega}}\left(e^{q \omega}-1\right)^{1 / q}\left(\int_{t-n}^{t-n+1}\left\|g_{2}(s)\right\|^{p} d s\right)^{1 / p} \\
& \leq \frac{M e^{-\omega n}}{\sqrt[q]{q \omega}}\left(e^{q \omega}+1\right)^{1 / q}\left(\int_{t-n}^{t-n+1}\left\|g_{2}(s)\right\|^{p} d s\right)^{1 / p} .
\end{aligned}
$$

Then, for $r>0$, we see that

$$
\begin{aligned}
& \frac{1}{\mu([-r, r])} \int_{[-r, r]}\left\|\psi_{n}(t)\right\| d \mu(t) \\
& \leq \frac{M e^{-\omega n}}{\sqrt[q]{q \omega}}\left(e^{q \omega}+1\right)^{1 / q} \frac{1}{\mu([-r, r])} \\
& \quad \times \int_{[-r, r]}\left(\int_{t-n}^{t-n+1}\left\|g_{2}(s)\right\|^{p} d s\right)^{1 / p} d \mu(t) .
\end{aligned}
$$

Since $g_{2}^{b} \in \epsilon\left(L^{p}(0,1 ; \mathbb{X}), \mu\right)$, the above inequality gives rise to $\psi_{n} \in$ $\epsilon(\mathbb{R}, \mathbb{X}, \mu)$. The above inequality also leads to

$$
\left\|\psi_{n}(t)\right\| \leq \frac{M e^{-\omega n}}{\sqrt[q]{q \omega}}\left(e^{q \omega}+1\right)^{1 / q}\left\|g_{2}\right\|_{S^{p}} .
$$

Since the series

$$
\frac{M}{\sqrt[q]{q \omega}}\left(e^{q \omega}+1\right)^{1 / q} \times \sum_{n=1}^{\infty} e^{-\omega n}
$$

is convergent, then we deduce from the Weierstrass M-test that the series $\sum_{n=1}^{\infty} \psi_{n}(t)$ is uniformly convergent on $\mathbb{R}$ and $\psi(t)=\int_{-\infty}^{t} S(t-$ 
$s) g_{2}(s) d s=\sum_{n=1}^{\infty} \psi_{n}(t)$. Applying $\psi_{n} \in \epsilon(\mathbb{R}, \mathbb{X}, \mu)$ and the inequality

$$
\begin{aligned}
& \frac{1}{\mu([-r, r])} \int_{[-r, r]}\|\psi(t)\| d \mu(t) \\
& \leq \frac{1}{\mu([-r, r])} \int_{[-r, r]}\left\|\psi(t)-\sum_{k=1}^{n} \psi_{k}(t)\right\| d \mu(t) \\
& \quad+\sum_{k=1}^{n} \frac{1}{\mu([-r, r])} \int_{[-r, r]}\left\|\psi_{k}(t)\right\| d \mu(t),
\end{aligned}
$$

we obtain the uniform limit $\psi(t)=\sum_{n=1}^{\infty} \psi_{n}(t) \in \epsilon(\mathbb{R}, \mathbb{X}, \mu)$. Therefore, $F(t)=\phi(t)+\psi(t)$ is $\mu$-pseudo almost automorphic. This ends of the proof.

Theorem 3.3. Let $\mu \in \mathcal{M}$ and $\beta>0, \alpha \neq 0$ with $\alpha+\beta>0$ and conditions (A1)-(A2) in Lemma 2.30 hold. Assume the conditions (H0), (T1)-(T2) are satisfied and the function $f=h_{1}+h_{2} \in P A A^{p}(\mathbb{R} \times$ $\mathbb{X}, \mathbb{X}, \mu)$ with $h_{1} \in A S^{p}(\mathbb{R} \times \mathbb{X}, \mathbb{X})$, and $h_{2}^{b} \in \epsilon\left(\mathbb{X}, L^{p}(0,1 ; \mathbb{X}), \mu\right)$. Then equation (1.1) has a unique $\mu$-pseudo almost automorphic mild solution on $\mathbb{R}$, provided that $\frac{M \mathbb{L}_{f}}{\omega}<1$.

Proof. Let $\Gamma: P A A(\mathbb{R}, \mathbb{X}, \mu) \rightarrow P A A(\mathbb{R}, \mathbb{X}, \mu)$ be the nonlinear operator defined by

$$
(\Gamma x)(t)=\int_{-\infty}^{t} S(t-s) f(s, x(s)) d s, \quad t \in \mathbb{R} .
$$

First, let us prove that $\Gamma(P A A(\mathbb{R}, \mathbb{X}, \mu)) \subseteq P A A(\mathbb{R}, \mathbb{X}, \mu)$. For each $x \in P A A(\mathbb{R}, \mathbb{X}, \mu)$, by using the fact that the range of an almost automorphic function is relatively compact combined with the above Lemma 2.21, Lemma 2.25 one can easily see that $f(\cdot, x(\cdot)) \in$ $P A A^{p}(\mathbb{R}, \mathbb{X}, \mu)$. Hence, from Lemma 3.2 , we know that $(\Gamma x)(\cdot) \in$ $P A A(\mathbb{R}, \mathbb{X}, \mu)$. That is, $\Gamma$ maps $P A A(\mathbb{R}, \mathbb{X}, \mu)$ into $P A A(\mathbb{R}, \mathbb{X}, \mu)$. Now, let us prove that $\Gamma$ has a unique fixed point. To the end, for each $t \in \mathbb{R}$, $x, y \in P A A(\mathbb{R}, \mathbb{X}, \mu)$, we have

$$
\begin{aligned}
\|(\Gamma x)(t)-(\Gamma y)(t)\| & \leq\left\|\int_{-\infty}^{t}\right\| S(t-s)[f(s, x(s))-f(s, y(s))] \| d s \\
& \leq M \int_{-\infty}^{t} e^{-\omega t}\|f(s, x(s))-f(s, y(s))\| d s
\end{aligned}
$$




$$
\begin{aligned}
& \leq M \mathbb{L}_{f} \int_{-\infty}^{t} e^{-\omega t}\|x(s)-y(s)\| d s \\
& \leq M \mathbb{L}_{f} \int_{-\infty}^{t} e^{-\omega t} d s\|x-y\|_{\infty} \\
& \leq \frac{M \mathbb{L}_{f}}{\omega}\|x-y\|_{\infty},
\end{aligned}
$$

which implies

$$
\|\Gamma x-\Gamma y\|_{\infty} \leq \frac{M \mathbb{L}_{f}}{\omega}\|x-y\|_{\infty} .
$$

Hence, by the Banach contraction principle with $\frac{M \mathbb{L}_{f}}{\omega}<1, \Gamma$ has a unique fixed-point $x$ in $P A A(\mathbb{R}, \mathbb{X}, \mu)$, which is the $\mu$-pseudo almost automorphic solution to equation (1.1). This finishes the proof.

Different Lipschitz type conditions involved in equation (1.1) are considered in the following results.

Theorem 3.4. Let $\mu \in \mathcal{M}$ and $\beta>0, \alpha \neq 0$ with $\alpha+\beta>$ 0 and conditions (A1)-(A2) in Lemma 2.30 be true. Assume that (H0), (T1), (T3) and (T5) hold, then equation (1.1) admits a unique $\mu$-pseudo almost automorphic mild solution whenever

$$
\left\|L_{f}\right\|_{S^{p}}<\frac{1-e^{-\omega}}{M}\left(\frac{\omega q}{1-e^{-\omega q}}\right)^{1 / q}
$$

where $\frac{1}{q}=1-\frac{1}{p}$.

Proof. Consider the nonlinear operator $\Gamma$ given by

$$
(\Gamma x)(t)=\int_{-\infty}^{t} S(t-s) f(s, x(s)) d s, \quad t \in \mathbb{R} .
$$

Letting $x \in P A A(\mathbb{R}, \mathbb{X}, \mu)$, with Lemma 2.21 and Lemma 2.26 it follows that the function $s \rightarrow f(s, x(s))$ is in $P A A^{p}(\mathbb{R}, \mathbb{X}, \mu)$. Moreover, from Lemma 3.2, we infer that $\Gamma x \in P A A(\mathbb{R}, \mathbb{X}, \mu)$, that is, $\Gamma$ maps $P A A(\mathbb{R}, \mathbb{X}, \mu)$ into itself. Next, we prove that the operator $\Gamma$ has a unique fixed point in $P A A(\mathbb{R}, \mathbb{X}, \mu)$. Indeed, for each $t \in \mathbb{R}$, $x, y \in P A A(\mathbb{R}, \mathbb{X}, \mu)$, we have

$$
\|\Gamma x(t)-\Gamma y(t)\| \leq\left\|\int_{-\infty}^{t} S(t-s)[f(s, x(s))-f(s, y(s))] d s\right\|
$$




$$
\begin{aligned}
& \leq M \int_{-\infty}^{t} e^{-\omega t}\|f(s, x(s))-f(s, y(s))\| d s \\
& \leq M \int_{-\infty}^{t} e^{-\omega t} L_{f}(s) d s\|x-y\|_{\infty} \\
& =\sum_{n=1}^{\infty} \int_{t-n}^{t-n+1} M e^{-\omega t} L_{f}(s) d s\|x-y\|_{\infty} \\
& \leq \sum_{n=1}^{\infty}\left(\int_{t-n}^{t-n+1} M^{q} e^{-\omega q t} d s\right)^{1 / q}\left\|L_{f}\right\|_{S^{p}}\|x-y\|_{\infty} \\
& \leq \frac{M}{1-e^{-\omega}}\left(\frac{1-e^{-q \omega}}{\omega q}\right)^{1 / q}\left\|L_{f}\right\|_{S^{p}}\|x-y\|_{\infty} .
\end{aligned}
$$

Thus, we get

$$
\|\Gamma x-\Gamma y\|_{\infty} \leq \frac{M}{1-e^{-\omega}}\left(\frac{1-e^{-q \omega}}{\omega q}\right)^{1 / q}\left\|L_{f}\right\|_{S^{p}}\|x-y\|_{\infty} .
$$

Since

$$
\left\|L_{f}\right\|_{S^{p}}<\frac{1-e^{-\omega}}{M}\left(\frac{\omega q}{1-e^{-\omega q}}\right)^{1 / q},
$$

$\Gamma$ has a unique fixed point $x \in P A A(\mathbb{R}, \mathbb{X}, \mu)$ by the Banach contraction principle. The proof is finished.

Theorem 3.5. Let $\mu \in \mathcal{M}, \beta>0, \alpha \neq 0$ with $\alpha+\beta>0$ and conditions (A1)-(A2) in Lemma 2.30 hold. Assume that (H0), (T1) and (T4) are true. Then there exists a unique $\mu$-pseudo almost automorphic mild solution to equation (1.1), provided that

$$
\frac{M}{1-e^{-\omega}}\left\|\mathcal{L}_{f}\right\|_{S^{\mathrm{r}}}<1 .
$$

Proof. Consider the nonlinear operator $\Gamma$ given by

$$
(\Gamma x)(t)=\int_{-\infty}^{t} S(t-s) f(s, x(s)) d s, \quad t \in \mathbb{R} .
$$

Letting $x \in P A A(\mathbb{R}, \mathbb{X}, \mu)$, with Lemma 2.21 and Theorem 2.28, it follows that the function $s \rightarrow f(s, x(s))$ is in $P A A^{\bar{q}}(\mathbb{R}, \mathbb{X}, \mu), \bar{q} \in[1, p)$. Moreover, from Lemma 3.2, we infer that $\Gamma x \in P A A(\mathbb{R}, \mathbb{X}, \mu)$, that is, $\Gamma$ maps $P A A(\mathbb{R}, \mathbb{X}, \mu)$ into itself. Next, we prove that the operator $\Gamma$ 
has a unique fixed point in $P A A(\mathbb{R}, \mathbb{X}, \mu)$. In fact, for each $t \in \mathbb{R}, x, y \in$ $P A A(\mathbb{R}, \mathbb{X}, \mu)$, we have

$$
\begin{aligned}
\|\Gamma x(t)-\Gamma y(t)\| & \leq\left\|\int_{-\infty}^{t} S(t-s)[f(s, x(s))-f(s, y(s))] d s\right\| \\
& \leq M \int_{-\infty}^{t} e^{-\omega t}\|f(s, x(s))-f(s, y(s))\| d s \\
& \leq M \int_{-\infty}^{t} e^{-\omega t} \mathcal{L}_{f}(s)\|x(s)-y(s)\| d s \\
& \leq \sum_{n=1}^{\infty} M e^{-\omega(n-1)} \int_{t-n}^{t-n+1} \mathcal{L}_{f}(s)\|x-y\|_{\infty} d s \\
& \leq \sum_{n=1}^{\infty} M e^{-\omega(n-1)}\left(\int_{t-n}^{t-n+1}\left\|\mathcal{L}_{f}\right\|_{S^{\mathrm{r}}} d s\right)^{1 / \mathrm{r}}\|x-y\|_{\infty} \\
& \leq \frac{M}{1-e^{-\omega}}\left\|\mathcal{L}_{f}\right\|_{S^{\mathrm{r}}}\|x-y\|_{\infty},
\end{aligned}
$$

which gives

$$
\|\Gamma x-\Gamma y\|_{\infty} \leq \frac{M}{1-e^{-\omega}}\left\|\mathcal{L}_{f}\right\|_{S^{\mathrm{x}}}\|x-y\|_{\infty} .
$$

In view of the inequality

$$
\frac{M}{1-e^{-\omega}}\left\|\mathcal{L}_{f}\right\|_{S^{\mathrm{r}}}<1
$$

$\Gamma$ has a unique fixed point $x \in P A A(\mathbb{R}, \mathbb{X}, \mu)$ due to the Banach contraction principle. The proof is completed.

We next deal with existence of $\mu$-pseudo almost automorphic solutions to equation (1.1) when the forcing term $f$ does not necessarily satisfy Lipschitz type conditions. The following existence result is based upon Leray-Schauder nonlinear alternative theorem. For that, we require the following assumption:

(T7) There exists a continuous nondecreasing function $\Theta:[0, \infty) \rightarrow$ $(0, \infty)$ such that

$$
\|f(t, \theta)\| \leq \Theta(\|\theta\|) \quad \text { for all } t \in \mathbb{R} \text { and } \theta \in \mathbb{X}
$$


Theorem 3.6. Let $\mu \in \mathcal{M}$ and $\beta>0, \alpha \neq 0$ with $\alpha+\beta>0$ and conditions (A1)-(A2) in Lemma 2.30 hold. Assume the conditions $(\mathrm{H} 0),(\mathrm{T} 1)$ are satisfied. Let $f: \mathbb{R} \times \mathbb{X} \rightarrow \mathbb{X}$ be a function which satisfies assumptions (T5)-(T7) and the following additional conditions:

(i) For each $\kappa \geq 0$, the function $t \rightarrow \int_{-\infty}^{t} M e^{-\omega(t-s)} \Theta(\kappa \varrho(s)) d s$ belongs to $B C(\mathbb{R})$. We set

$$
\lambda(\kappa)=M\left\|\int_{-\infty}^{t} e^{-\omega(t-s)} \Theta(\kappa \varrho(s)) d s\right\|_{\varrho}
$$

(ii) For each $\varepsilon>0$, there is a $\delta>0$ such that, for every $x, y \in C_{\varrho}(\mathbb{X})$, $\|x-y\|_{\varrho} \leq \delta$ impliesx that

$$
\int_{-\infty}^{t} M e^{-\omega(t-s)}\|f(s, x(s))-f(s, y(s))\| d s \leq \varepsilon
$$

for all $t \in \mathbb{R}$.

(iii) $\liminf _{\xi \rightarrow \infty} \frac{\xi}{\lambda(\xi)}>1$.

(iv) For all $a, b \in \mathbb{R}, a<b$ and $\kappa>0$, the set $\{f(s, x): a \leq s \leq b$, $\left.x \in C_{\varrho}(\mathbb{X}),\|x\|_{\varrho} \leq \kappa\right\}$ is relatively compact in $\mathbb{X}$.

Then equation (1.1) has at least one $\mu$-pseudo almost automorphic mild solution on $t \in \mathbb{R}$.

Proof. We define the nonlinear operator $\Gamma: C_{\varrho}(\mathbb{X}) \rightarrow C_{\varrho}(\mathbb{X})$ by

$$
(\Gamma x)(t):=\int_{-\infty}^{t} S(t-s) f(s, x(s)) d s, \quad t \in \mathbb{R} .
$$

We will show that $\Gamma$ has a fixed point in $P A A(\mathbb{R}, \mathbb{X}, \mu)$. For the sake of convenience, we divide the proof into several steps.

(i) For $x \in C_{\varrho}(\mathbb{X})$, we have that

$$
\begin{aligned}
\|(\Gamma x)(t)\| & \leq \int_{-\infty}^{t} M e^{-\omega(t-s)} \Theta(\|x(s)\|) d s \\
& \leq \int_{-\infty}^{t} M e^{-\omega(t-s)} \Theta\left(\|x\|_{\varrho} \varrho(s)\right) d s .
\end{aligned}
$$

It follows from condition (i) that $\Gamma$ is well defined.

(ii) The operator $\Gamma$ is continuous. In fact, for any $\varepsilon>0$, we take $\delta>0$ involved in condition (ii). If $x, y \in C_{\varrho}(\mathbb{X})$ and $\|x-y\|_{\varrho} \leq \delta$, 
then

$$
\begin{aligned}
& \|(\Gamma x)(t)-(\Gamma y)(t)\| \\
& \leq \int_{-\infty}^{t} M e^{-\omega(t-s)}\|f(s, x(s))-f(s, y(s))\| d s \leq \varepsilon,
\end{aligned}
$$

which shows the assertion.

(iii) We will show that $\Gamma$ is completely continuous. We set $B_{\kappa}(\mathbb{X})$ for the closed ball with center at 0 and radius $\kappa$ in the space $\mathbb{X}$. Let $V=\Gamma\left(B_{\kappa}\left(C_{\varrho}(\mathbb{X})\right)\right)$ and $\nu=\Gamma(x)$ for $x \in B_{\kappa}\left(C_{\varrho}(\mathbb{X})\right)$. First, we will prove that $V(t)$ is a relatively subset of $\mathbb{X}$ for each $t \in \mathbb{R}$. It follows from condition (i) that the function $s \rightarrow M e^{-\omega s} \Theta(\kappa \varrho(t-s))$ is integrable on $[0, \infty)$. Hence, for $\varepsilon>0$, we can choose $a \geq 0$ such that $\int_{a}^{\infty} M e^{-\omega s} \Theta(\kappa \varrho(t-s)) d s \leq \varepsilon$. Since

$$
\nu(t)=\int_{0}^{a} S(s) f(t-s, x(t-s)) d s+\int_{a}^{\infty} S(s) f(t-s, x(t-s)) d s
$$

and

$$
\left\|\int_{a}^{\infty} S(s) f(t-s, x(t-s)) d s\right\| \leq \int_{a}^{\infty} M e^{-\omega s} \Theta(\kappa \varrho(t-s)) d s \leq \varepsilon,
$$

we get $\nu(t) \in a \overline{c_{0}(\mathbb{K})}+B_{\varepsilon}(\mathbb{X})$, where $c_{0}(\mathbb{K})$ denotes the convex hull of $\mathbb{K}$ and

$$
\mathbb{K}=\left\{S(s) f(\xi, x): 0 \leq s \leq a, t-a \leq \xi \leq t,\|x\|_{\varrho} \leq \kappa\right\} .
$$

Considering the strong continuity of $S(\cdot)$ and condition (iv) on $f$, we see that $\mathbb{K}$ is a relatively compact set, and $V(t) \subseteq a \overline{c_{0}(\mathbb{K})}+$ $B_{\varepsilon}(\mathbb{X})$, which establishes our assertion.

Second, we show that the set $V$ is equicontinuous. In fact, we can decompose

$$
\begin{aligned}
& \nu(t+s)-\nu(t) \\
& =\int_{0}^{s} S(\sigma) f(t+s-\sigma, x(t+s-\sigma)) d \sigma \\
& \quad+\int_{0}^{a}[S(\sigma+s)-S(\sigma)] f(t-\sigma, x(t-\sigma)) d \sigma \\
& \quad+\int_{a}^{\infty}[S(\sigma+s)-S(\sigma)] f(t-\sigma, x(t-\sigma)) d \sigma .
\end{aligned}
$$


For each $\varepsilon>0$, we can choose $a>0$ and $\delta_{1}>0$ such that

$$
\begin{aligned}
& \| \int_{0}^{s} S(\sigma) f(t+s-\sigma, x(t+s-\sigma)) d \sigma \\
& \quad+\int_{a}^{\infty}[S(\sigma+s)-S(\sigma)] f(t-\sigma, x(t-\sigma) d \sigma \| \\
& \leq \int_{0}^{s} M e^{-\omega \sigma} \Theta(\kappa \varrho(t+s-\sigma)) d \sigma \\
& \quad+\int_{a}^{\infty}\left[M\left(e^{-\omega(\sigma+s)}+e^{-\omega \sigma}\right)\right] \Theta(\kappa \varrho(t-\sigma)) d \sigma \\
& \leq \frac{\varepsilon}{2}
\end{aligned}
$$

for $s \leq \delta_{1}$. Moreover, since $\{f(t-\sigma, x(t-\sigma)): 0 \leq \sigma \leq a, x \in$ $\left.B_{\kappa}\left(C_{\varrho}(\mathbb{X})\right)\right\}$ is relatively compact and $S(\cdot)$ is strongly continuous, we can choose $\delta_{2}>0$ such that

$$
\|[S(\sigma+s)-S(\sigma)] f(t-\sigma, x(t-\sigma))\| \leq \frac{\varepsilon}{2 a} \quad \text { for } s \leq \delta_{2} .
$$

Combining these estimates, we get $\|\nu(t+s)-\nu(t)\| \leq \varepsilon$ for $s$ small enough and independent of $x \in B_{\kappa}\left(C_{\varrho}(\mathbb{X})\right)$.

Finally, in view of condition (i), it is easy to see that

$$
\frac{\|\nu(t)\|}{\varrho(t)} \leq \frac{1}{\varrho(t)} \int_{-\infty}^{t} M e^{-\omega(t-s)} \Theta(\kappa \varrho(s)) d s \longrightarrow 0, \quad|t| \rightarrow \infty,
$$

and this convergence is independent of $x \in B_{\kappa}\left(C_{\varrho}(\mathbb{X})\right)$. Hence, by Lemma $2.32, V$ is a relatively compact set in $C_{\varrho}(\mathbb{X})$.

(iv) Let us now assume that $x^{\gamma}(\cdot)$ is a solution of equation $x^{\gamma}=$ $\gamma \Gamma\left(x^{\gamma}\right)$ for some $0<\gamma<1$. We can estimate

$$
\begin{aligned}
\left\|x^{\gamma}(t)\right\| & =\gamma\left\|\int_{-\infty}^{t} S(t-s) f\left(s, x^{\gamma}(s)\right)\right\| \\
& \leq \int_{-\infty}^{t} M e^{-\omega(t-s)} \Theta\left(\left\|x^{\gamma}\right\|_{\varrho} \varrho(s)\right) d s \\
& \leq \lambda\left(\left\|x^{\gamma}\right\|_{\varrho}\right) \varrho(t) .
\end{aligned}
$$

Hence, we get

$$
\frac{\left\|x^{\gamma}\right\|_{\varrho}}{\lambda\left(\left\|x^{\gamma}\right\|_{\varrho}\right)} \leq 1
$$


and, combining with condition (iii), we conclude that the set $\left\{x^{\gamma}: x^{\gamma}=\gamma \Gamma\left(x^{\gamma}\right), \gamma \in(0,1)\right\}$ is bounded.

(v) It follows from Lemma 2.21, (T6)-(T7) and Lemma 2.27 that the function $t \rightarrow f(t, x(t))$ belongs to $P A A^{p}(\mathbb{R}, \mathbb{X}, \mu)$, whenever $x \in P A A(\mathbb{R}, \mathbb{X}, \mu)$. Moreover, from Lemma 3.2, we infer that $\Gamma(P A A(\mathbb{R}, \mathbb{X}, \mu)) \subseteq P A A(\mathbb{R}, \mathbb{X}, \mu)$ and noting that $P A A(\mathbb{R}, \mathbb{X}, \mu)$ is a closed subspace of $C_{\varrho}(\mathbb{X})$, consequently, we can consider $\Gamma: P A A(\mathbb{R}, \mathbb{X}, \mu) \rightarrow P A A(\mathbb{R}, \mathbb{X}, \mu)$. Using properties (i)-(iii), we deduce that this map is completely continuous. Applying Lemma 2.33, we infer that $\Gamma$ has a fixed point $x \in P A A(\mathbb{R}, \mathbb{X}, \mu)$, which completes the proof.

The following result is a direct consequence of Theorem 3.6.

Corollary 3.7. Let $\beta>0, \alpha \neq 0$ with $\alpha+\beta>0$ and conditions (A1)-(A2) in Lemma 2.30 hold. Assume that (H0) and (T1) are satisfied. Let $f: \mathbb{R} \times \mathbb{X} \rightarrow \mathbb{X}$ be a function satisfying assumptions (T5)-(T6) and the following condition

$$
\|f(t, x)-f(t, y)\| \leq \zeta\|x-y\|^{\iota}, \quad 0<\iota<1
$$

for all $t \in \mathbb{R}$ and $x, y \in \mathbb{X}$, where $\zeta>0$ is a constant. Moreover, assume the following conditions hold:

(a) $f(t, 0)=q$.

(b) $\sup _{t \in \mathbb{R}} \int_{-\infty}^{t} M e^{-\omega(t-s)} \varrho(s)^{\iota} d s=\zeta_{1}<\infty$.

(c) For all $a, b \in \mathbb{R}, a<b$, and $\kappa>0$, the set $\{f(s, x): a \leq s \leq b, x \in$ $\left.C_{\varrho}(\mathbb{X}),\|x\|_{\varrho} \leq \kappa\right\}$ is relatively compact in $\mathbb{X}$.

Then equation (1.1) has a $\mu$-pseudo almost automorphic mild solution on $\mathbb{R}$.

Proof. Let $\zeta_{0}=\|q\|, \zeta_{2}=\zeta$, and $\Theta(\theta)=\zeta_{0}+\zeta_{2} \theta^{\iota}$. Thus, condition (T7) is true. In view of assumption (b), we can see that condition (i) in Theorem 3.6 is also true. To prove condition (ii) in Theorem 3.6, note that for each $\varepsilon>0$ there exists $0<\delta^{\iota}<$ $\frac{\varepsilon}{\zeta_{1} \zeta_{2}}$ such that, for every $x, y \in C_{\varrho}(\mathbb{X}),\|x-y\|_{\varrho} \leq \delta$ implies that $\int_{-\infty}^{t} e^{-\omega(t-s)}\|f(s, x(s))-f(s, y(s))\| d s \leq \varepsilon$ for all $t \in \mathbb{R}$. On the other hand, hypothesis (iii) in Theorem 3.6 can be easily verified by the definition of $\Theta$. This completes the proof. 
Remark 3.8. Condition (T7) in Theorem 3.6 can be replaced by the following more general condition:

(T8) There exist a bounded continuous function $\mathbb{p}(t): \mathbb{R} \rightarrow(0,+\infty)$ and a continuous nondecreasing function $\Theta:[0, \infty) \rightarrow(0, \infty)$ such that

$$
\|f(t, \theta)\| \leq \mathbb{p}(t) \Theta(\|\theta\|) \text { for all } t \in \mathbb{R} \text { and } \theta \in \mathbb{X} .
$$

In fact, if we let $\mathbb{P}=\sup _{t \in \mathbb{R}}|\mathfrak{p}(t)|, \Theta(\|\theta\|):=\mathbb{P} \cdot \Theta(\|\theta\|)$, then the main proofs of Theorem 3.6 still hold true.

Example 3.9. Consider the following problem:

$$
\left\{\begin{aligned}
\frac{\partial u}{\partial t}(t, x)= & \frac{\partial^{2} u}{\partial x^{2}}(t, x)+\int_{-\infty}^{t} e^{-(t-s)} \frac{\partial^{2} u}{\partial x^{2}}(s, x) d s \\
& +f(t, u(t, x)), \quad t \in \mathbb{R}, \\
u(0, t)= & u(\pi, t)=0,
\end{aligned}\right.
$$

where $f \in L^{2}[0, \pi] \rightarrow L^{2}[0, \pi]$ is given by

$$
f(t, u(t, x))=u(t, x) \sin \frac{1}{2+\cos t+\cos \pi t}+e^{-|t|} \sin u(t, x) .
$$

We set $X:=L^{2}[0, \pi]$, and define $A:=\frac{\partial^{2} u}{\partial x^{2}}$, with the domain of the operator $A$ as

$$
D(A):=\left\{u \in L^{2}[0, \pi]: u(0)=u(\pi)=0, u^{\prime \prime} \in L^{2}[0, \pi]\right\} .
$$

From the deduction of [22, Example 4.10], we know that the operator $A$ satisfies condition (A2) in Lemma 2.30 and generates an immediately norm continuous and compact $C_{0}$-semigroup $T(t)$ on $\mathbb{X}$. Thus, the problem (3.1) can be converted into the abstract system (1.1) with $\alpha=\beta=1$.

If we define its Radon-Nikodym derivative as $\frac{d \mu(t)}{d t}:=1+t^{2}$, then the measure $\mu$ is absolutely continuous with respect to the Lebesgue measure $([\mathbf{3}, \mathbf{7}])$. Note that the function $f$ defined above is Stepanov type $\mu$-pseudo almost automorphic, and

$$
\|f(t, u)-f(t, v)\| \leq 2\|u-v\| .
$$

By a consequence of Theorem 3.3, we conclude that the problem (3.1) admits a unique $\mu$-pseudo almost automorphic solution if $\frac{M}{\omega}<\frac{1}{2}$. 


\section{REFERENCES}

1. S. Abbas, Pseudo almost automorphic solutions of some nonlinear integrodifferential equations, Comp. Math. Appl. 62 (2011), 2259-2272.

2. S. Abbas, Y.K. Chang and M. Hafayed, Stepanov type weighted pseudo almost automorphic sequences and their applications to difference equations, Nonlin. Stud. 21 (2014), 99-111.

3. J. Blot, P. Cieutat and K. Ezzinbi, Measure theory and pseudo almost automorphic functions: New development and applications, Nonlin. Anal. $\mathbf{7 5}$ (2012), 2426-2447.

4. S. Bochner, A new approach to almost automorphicity, Proc. Nat. Acad. Sci. 48 (1962), 2039-2043.

5. A. Caicedo, C. Cuevas, G. Mophou and G.M. N'Guérékata, Asymptotic behavior of solutions of some semilinear functional differential and integro-differential equations with infinite delay in Banach spaces, J. Franklin Inst. 349 (2012), 1-24.

6. Y.K. Chang, X.X. Luo and G.M. N'Guérékata, Asymptotically typed solutions to a semilinear integral equation, J. Integral Equations Appl. 26 (2014), 323-343.

7. Y.K. Chang, G.M. N'Guérékata and R. Zhang, Stepanov-like weighted pseudo almost automorphic functions via measure theory, Bull. Malays. Math. Sci. Soc. (2015), doi:10.1007/s40840-015-0206-1.

8. Y.K. Chang, M.J. Zhang and R. Ponce, Weighted pseudo almost automorphic solutions to a semilinear fractional differential equation with Stepanov-like weighted pseudo almost automorphic nonlinear term, Appl. Math. Comp. 257 (2015), 158168.

9. Y.K. Chang, R. Zhang, G.M. N'Guérékata, Weighted pseudo almost automorphic solutions to nonautonomous semilinear evolution equations with delay and $S^{p}$-weighted pseudo almost automorphic coefficients, Top. Meth. Nonlin. Anal. 43 (2014), 69-88.

10. T. Diagana, Existence of pseudo-almost automorphic solutions to some abstract differential equations with $S^{p}$-pseudo-almost automorphic coefficients, Nonlin. Anal. 70 (2009), 3781-3790.

11. , Almost automorphic type and almost periodic type functions in abstract spaces, Springer, New York, 2013.

12. T. Diagana, G.M. Mophou and G.M. N'Guérékata, Existence of weighted pseudo almost periodic solutions to some classes of differential equations with $S^{p}$. weighted pseudo almost periodic coefficients, Nonlin. Anal. 72 (2010), 430-438.

13. H.S. Ding, J. Liang and T.J. Xiao, Almost automorphic solutions to nonautonomous semilinear evolution equations in Banach spaces, Nonlin. Anal. 73 (2010), 1426-1438.

14. H.S. Ding, W. Long and G.M. N'Guérékata, A composition theorem for weighted pseudo-almost automorphic functions and applications, Nonlin. Anal. 73 (2010), 2644-2650.

15. Z.B. Fan, J. Liang and T.J. Xiao, On Stepanov-like (pseudo) almost automorphic functions, Nonlin. Anal. 74 (2011), 2853-2861. 
16. A. Granas and J. Dugundji, Fixed point theory, Springer-Verlag, New York, 2003.

17. H.R. Henríquez and C. Cuevas, Almost automorphy for abstract neutral differential equation via control theory, Ann. Matem. 192 (2013), 393-405.

18. H.R. Henríquez and C. Lizama, Compact almost automorphic solutions to integral equations with infinite delay, Nonlin. Anal. 71 (2009), 6029-6037.

19. V. Kavitha, S. Abbas and R. Murugesuz, Asymptotically almost automorphic solutions of fractional order neutral integro-differential equations, Bull. Malays. Math. Sci. Soc., accepted.

20. J. Liang, G.M. N'Guérékata and T.J. Xiao, et al., Some properties of pseudo almost automorphic functions and applications to abstract differential equations, Nonlin. Anal. 70 (2009), 2731-2735.

21. J. Liang, J. Zhang and T.J. Xiao, Composition of pseudo almost automorphic and asymptotically almost automorphic functions, J. Math. Anal. Appl. 340 (2008), 1493-1499.

22. C. Lizama and R. Ponce, Bounded solutions to a class of semilinear integrodifferential equations in Banach spaces, Nonlin. Anal. 74 (2011), 3397-3406.

23. I. Mishra and D. Bahuguna, Weighted pseudo almost automorphic solution of an integro-differential equation with weighted Stepanov-like pseudo almost automorphic forcing term, Appl. Math. Comp. 219 (2013), 5345-5355.

24. G.M. Mophou, Weighted pseudo almost automorphic mild solutions to semilinear fractional differential equations, Appl. Math. Comp. 217 (2011), 75797587.

25. G.M. N'Guérékata, Almost automorphic and almost periodic functions in abstract spaces, Kluwer Academic, New York, 2001.

26. , Topics in almost automorphy, Springer, New York, 2005.

27. G.M. N'Guérékata and A. Pankov, Stepanov-like almost automorphic functions and monotone evolution equations, Nonlin. Anal. 68 (2008), 2658-2667.

28. R. Ponce, Bounded mild solutions to fractional integro-differential equations in banach spaces, Semigroup Forum 87 (2013), 377-392.

29. J.R. Wang, Y. Zhou and M. Medved, On the solvability and optimal controls of fractional integrodifferential evolution systems with infinite delay, J. Optim. Theory Appl. 152 (2012), 31-50.

30. Z.N. Xia, Weighted pseudo almost automorphic solutions of hyperbolic semilinear integrodifferential equations, Nonlin. Anal. 95 (2014), 50-65.

31. T.J. Xiao, J. Liang and J. Zhang, Pseudo almost automorphic solutions to semilinear differential equations in Banach space, Semigroup Forum 76 (2008), $518-524$.

32. R. Zhang, Y.K. Chang and G.M. N'Guérékata, New composition theorems of Stepanov-like weighted pseudo almost automorphic functions and applications to nonautonomous evolution equations, Nonlin. Anal. RWA 13 (2012), 2866-2879. 
33. R. Zhang, Y.K. Chang and G.M. N'Guérékata, Weighted pseudo almost automorphic solutions for non-autonomous neutral functional differential equations with infinite delay, Sci. Sinica Math. 43 (2013), 273-292, doi: 10.1360/012013-9 (in Chinese).

34. W Weighted pseudo almost automorphic mild solutions to semilinear integral equations with $S^{p}$-weighted pseudo almost automorphic coefficients, Discr. Cont. Dyn. Syst. 33 (2013), 5525-5537.

School of Mathematics and Statistics, Xidian University, Xi'an 710071 , P.R. China

Email address: lzchangyk@163.com

Department of Mathematics, Lanzhou Jiaotong University, Lanzhou 730070 , P.R. CHINA

Email address: Lzweixy0931@163.com

Department of Mathematics, Morgan State University, 1700 E. Cold Spring Lane, Baltimore, MD 21251

Email address: nguerekata@aol.com 